\title{
Segmentation in Noisy Medical Images Using PCA Model Based Particle Filtering
}

\author{
Wei $\mathrm{Qu}^{a}$, Xiaolei Huang ${ }^{b}$, and Yuanyuan $\mathrm{Jia}^{c}$ \\ ${ }^{a}$ Siemens Medical Solutions USA Inc., AX Division, Hoffman Estates, IL 60192; \\ ${ }^{b}$ Computer Science and Engineering Department, Lehigh University, Bethlehem, PA 18015; \\ ${ }^{c}$ Bioengineering Department, University of Illinois at Chicago, Chicago, IL 60607 \\ Emails: weiqu@ siemens.com ${ }^{a}$, huang@cse.lehigh.edu ${ }^{b}$, yjia2@ uic.edu ${ }^{c}$
}

\begin{abstract}
Existing common medical image segmentation algorithms such as snake or graph cut usually could not generate satisfying results for noisy medical images such as X-ray angiographical and ultrasound images where the image quality is very poor including substantial background noise, low contrast, clutter, etc. In this paper, we present a robust segmentation method for noisy medical image analysis using Principle Component Analysis (PCA) based particle filtering. It exploits the prior clinical knowledge of desired object's shape through a PCA model. The preliminary results have shown the effectiveness and efficiency of the proposed approach on both synthetic and real clinical data.
\end{abstract}

Keywords: Medical image segmentation, Particle filter, Principle component analysis, Medical image processing.

\section{INTRODUCTION}

This work is motivated by the need to perform reliable and rapid segmentation in noisy medical images such as X-ray and ultrasound images. The applications include the widely used X-ray angiographic left ventricular analysis, cardia segmentation in ultrasonic images, among others. In these applications, imaging conditions can not be optimized for each frame. The images have to be processed notwithstanding clutter, noise, and low contrast. Furthermore, these images are usually subject to nonuniform illumination, fadeout, glare, and loss of focus.
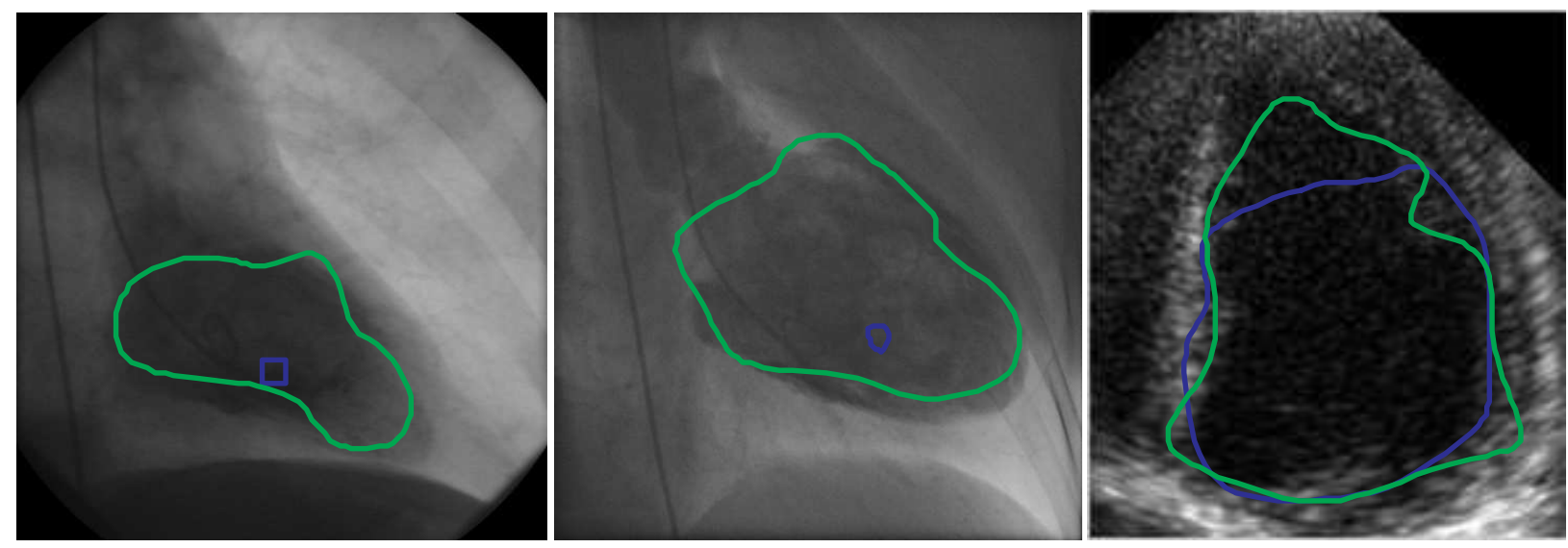

Figure 1. Segmentation result using graph cut (blue curve) and snake (green curve). 
Intensive research has been devoted to robust medical image interpretation in the literature. Thresholding, Region Growing, Edge detection and grouping, Markov Random Fields (MRF), ${ }^{1}$ active contour or deformable models, ${ }^{2}$ level sets, ${ }^{3,4}$ Graph Cut, ${ }^{5-7}$ and Mean shift ${ }^{8}$ are the several common mathematical models for segmentation. Significant extensions and integrations of these frameworks ${ }^{9-14}$ have been developed over the years to improve their efficiency, applicability and accuracy. Several algorithms above, including thresholding, region growing, edge detection, and Mean Shift require the setting of threshold or bandwidth parameters whose optimal values are difficult to choose in X-ray or ultrasound images. Level sets are geometric models that can partition an entire image frame into regions of smooth interiors; level set computation is often expensive however and its topology-free property makes it difficult to obtain coherent object boundaries in noisy images such as X-ray and ultrasound. Markov Random Fields (MRF) is a region-based method that groups pixels whose intensities follow consistent statistics, thus it is less sensitive to localized image noise than edges. MRF may fail to separate foreground and background in the presence of intensity inhomogeneity and it often generates small holes/islands inside the segmented object. Active contours (or "snakes", deformable models) are parametric curves or surfaces that deform under the influence of internal smoothness and external image forces to delineate object boundary. Compared to local edge-based methods, deformable models have the advantage of estimating boundary with smooth curves or surfaces that bridge over boundary gaps, although they suffer from the well known local minima problem so that if a model is initialized far away from the boundary, it can be trapped by spurious edges. Graph cut tackles the segmentation problem by graph theory and aims to partition an image by removing edges connecting pixels with dissimilar color/intensity. Graph cut addresses the local minima problem to some extent but its results are sensitive to seed-point initialization and it may fail at low-contrast boundaries. Figure 1 illustrates some of the problems using snakes and graph cut. As one can see, snake-based algorithms and graph cut approaches could not generate satisfying results for the X-ray and ultrasound images due to the poor image quality and/or tremendous background noise.

Because of the challenging nature of the medical image segmentation problem, statistical model based segmentation approaches such as Active Shape Models (ASM ) and Active Appearance Models (AAM) ${ }^{15-18}$ are widely adopted. An ASM is a statistical shape model learned a priori from examples to capture variations in the shape of an object of interest in certain kinds of medical images. When applied to segmentation, the models deform toward object boundary but with constraints to deform only in ways characteristic of the object they represent. These statistical models encode high-level knowledge in a more specific manner and are often more robust for image interpretation. The boundary finding process in ASMs is deterministic, however, which requires a model to be initialized sufficiently close to the object to converge and is sometimes prone to local minima.

In this paper, we propose a robust segmentation approach for noisy medical image analysis using PCA-based particle filtering. It exploits the prior clinical knowledge of the desired object's shape through a PCA model. To fit the PCA model to image data, the basic idea is to use particle filters to sample the shape transformation and constrained deformation space, evaluate the likelihood of each sample shape given observed image data, and estimate the object shape by weighted combination of all shape samples (particles). The stochastic search, in comparison to ASM's deterministic search, is more effective in locating the global optimum for boundary interpretation, and therefore allows fully automated segmentation without manual model-pose initialization. Preliminary experimental results using both synthetic data and a real X-ray left ventricular angiography image sequence have demonstrated the superior performance of the proposed algorithm.

\section{SEGMENTATION USING PCA-BASED PARTICLE FILTERING}

\subsection{System Model}

We use graphical model to analyze the segmentation in noisy medical images. Fig. 2(a) shows our static graphical model. It has three layers: PCA shape space, pixel state space, and image observation space. The 


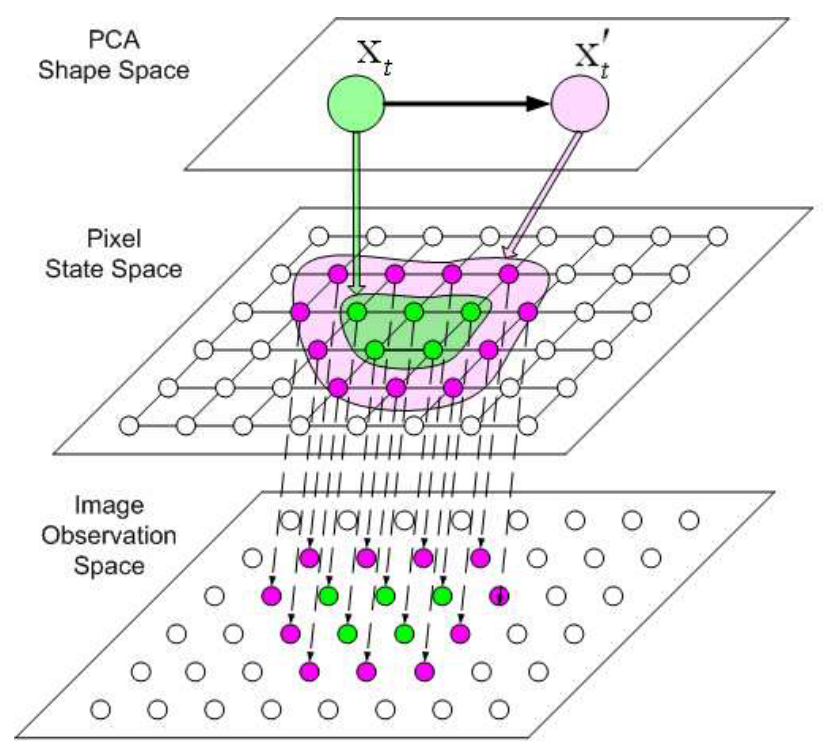

(a)

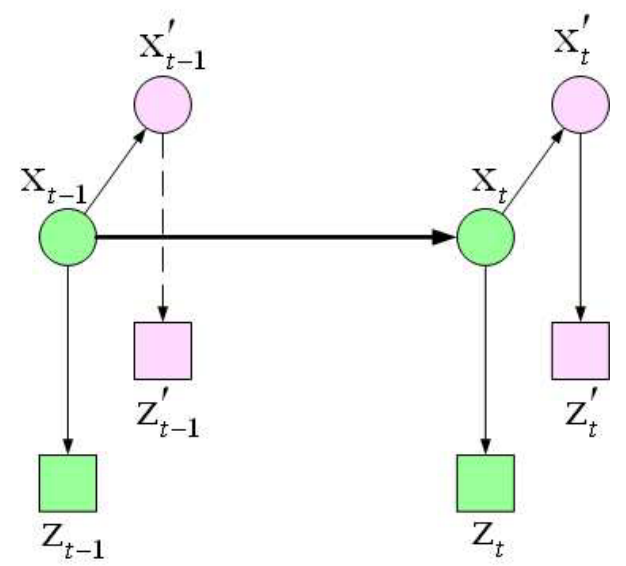

(b)

Figure 2. Graphical model of the proposed medical image segmentation framework. (a) Static graphical model; (b) Dynamic graphical model.

bottom layer models the actual image observations where each circle node represents a pixel. Since we assume the observed pixels are independent, there is no link among the nodes. The middle layer models the state of each pixel in the image data. The link between two nodes represents their correlation. The direct links between nodes in image observation space layer and the nodes in pixel state space layer represent the "generative" relationship. The green region in pixel state space models the desired object. In order to find a region to represent the background information and thus make the segmentation more stable, a belt region (purple belt in Fig. 2(a)) with predefined width around the desired object is selected. To decrease the dimensionality, we further introduce the Principle Component Analysis (PCA) shape space where two nodes represent the principle component vectors for object region and background belt region, respectively. The direct link between $\mathbf{x}_{t}$ and $\mathbf{x}_{t}^{\prime}$ indicate their correlation.

Since the object's contour is very difficult be estimated at one step but only after an iteration, we further propose a dynamic graphical model as illustrated in Fig. 2(b) where $t$ is the iteration index, $\mathbf{z}_{t}$ and $\mathbf{z}_{t}^{\prime}$ represent the pixel sets in image observation space for object and background belt regions, respectively. The middle layer is simplified based on graphical theory as the directed link from PCA shape space to pixel state space has the same direction with the link from pixel state space to image observation space.

\subsection{Particle Filtering Simulation}

We use particle filtering theory ${ }^{19,20}$ to simulate the proposed model. Specifically, the goal is to estimate the posterior density based on observations and the background belt region. With Bayes' rule, we can derive the 
updating rule for dynamic graphical model such as illustrated in Fig. 2(b)

$$
\begin{aligned}
& p\left(\mathbf{x}_{1: t} \mid \mathbf{x}_{1: t}^{\prime}, \mathbf{z}_{1: t}, \mathbf{z}_{1: t}^{\prime}\right) \\
= & \frac{p\left(\mathbf{x}_{t}, \mathbf{x}_{t}^{\prime}, \mathbf{z}_{t}, \mathbf{z}_{t}^{\prime} \mid \mathbf{x}_{1: t-1}, \mathbf{x}_{1: t-1}^{\prime}, \mathbf{z}_{1: t-1}, \mathbf{z}_{1: t-1}^{\prime}\right)}{p\left(\mathbf{x}_{t}^{\prime}, \mathbf{z}_{t}, \mathbf{z}_{t}^{\prime} \mid \mathbf{x}_{1: t-1}^{\prime}, \mathbf{z}_{1: t-1}, \mathbf{z}_{1: t-1}^{\prime}\right)} p\left(\mathbf{x}_{1: t-1} \mid \mathbf{x}_{1: t-1}^{\prime}, \mathbf{z}_{1: t-1}, \mathbf{z}_{1: t-1}^{\prime}\right) \\
= & \frac{p\left(\mathbf{z}_{t}, \mathbf{z}_{t}^{\prime} \mid \mathbf{x}_{t}, \mathbf{x}_{t}^{\prime}, \mathbf{x}_{1: t-1}, \mathbf{x}_{1: t-1}^{\prime}, \mathbf{z}_{1: t-1}, \mathbf{z}_{1: t-1}^{\prime}\right) p\left(\mathbf{x}_{t}, \mathbf{x}_{t}^{\prime} \mid \mathbf{x}_{1: t-1}, \mathbf{x}_{1: t-1}^{\prime}, \mathbf{z}_{1: t-1}, \mathbf{z}_{1: t-1}^{\prime}\right)}{p\left(\mathbf{x}_{t}^{\prime}, \mathbf{z}_{t}, \mathbf{z}_{t}^{\prime} \mid \mathbf{x}_{1: t-1}^{\prime}, \mathbf{z}_{1: t-1}, \mathbf{z}_{1: t-1}^{\prime}\right)} p\left(\mathbf{x}_{1: t-1} \mid \mathbf{x}_{1: t-1}^{\prime} \mathbf{z}_{1: t-1}, \mathbf{z}_{1: t}^{\prime}(2)\right. \\
= & \frac{p\left(\mathbf{z}_{t}, \mathbf{z}_{t}^{\prime} \mid \mathbf{x}_{t}, \mathbf{x}_{t}^{\prime}\right) p\left(\mathbf{x}_{t}, \mathbf{x}_{t}^{\prime} \mid \mathbf{x}_{t-1}\right)}{p\left(\mathbf{x}_{t}^{\prime}, \mathbf{z}_{t}, \mathbf{z}_{t}^{\prime} \mid \mathbf{x}_{1: t-1}^{\prime}, \mathbf{z}_{1: t-1}, \mathbf{z}_{1: t-1}^{\prime}\right)} p\left(\mathbf{x}_{1: t-1} \mid \mathbf{x}_{1: t-1}^{\prime}, \mathbf{z}_{1: t-1}, \mathbf{z}_{1: t-1}^{\prime}\right) \\
= & \frac{p\left(\mathbf{z}_{t} \mid \mathbf{z}_{t}^{\prime}, \mathbf{x}_{t}, \mathbf{x}_{t}^{\prime}\right) p\left(\mathbf{z}_{t}^{\prime} \mid \mathbf{x}_{t}, \mathbf{x}_{t}^{\prime}\right) p\left(\mathbf{x}_{t}^{\prime} \mid \mathbf{x}_{t}, \mathbf{x}_{t-1}\right) p\left(\mathbf{x}_{t} \mid \mathbf{x}_{t-1}\right)}{p\left(\mathbf{x}_{t}^{\prime}, \mathbf{z}_{t}, \mathbf{z}_{t}^{\prime} \mid \mathbf{x}_{1: t-1}^{\prime}, \mathbf{z}_{1: t-1}, \mathbf{z}_{1: t-1}^{\prime}\right)} p\left(\mathbf{x}_{1: t-1} \mid \mathbf{x}_{1: t-1}^{\prime}, \mathbf{z}_{1: t-1}, \mathbf{z}_{1: t-1}^{\prime}\right) \\
= & \frac{p\left(\mathbf{z}_{t} \mid \mathbf{x}_{t}\right) p\left(\mathbf{z}_{t}^{\prime} \mid \mathbf{x}_{t}^{\prime}\right) p\left(\mathbf{x}_{t}^{\prime} \mid \mathbf{x}_{t}\right) p\left(\mathbf{x}_{t} \mid \mathbf{x}_{t-1}\right)}{p\left(\mathbf{x}_{t}^{\prime}, \mathbf{z}_{t}, \mathbf{z}_{t}^{\prime} \mid \mathbf{x}_{1: t-1}^{\prime}, \mathbf{z}_{1: t-1}, \mathbf{z}_{1: t-1}^{\prime}\right)} p\left(\mathbf{x}_{1: t-1} \mid \mathbf{x}_{1: t-1}^{\prime}, \mathbf{z}_{1: t-1}, \mathbf{z}_{1: t-1}^{\prime}\right)
\end{aligned}
$$

In (2), we use the conditional independence properties from graphical model $p\left(\mathbf{z}_{t}, \mathbf{z}_{t}^{\prime} \mid \mathbf{x}_{t}, \mathbf{x}_{t}^{\prime}, \mathbf{x}_{1: t-1}, \mathbf{x}_{1: t-1}^{\prime}, \mathbf{z}_{1: t-1}, \mathbf{z}_{1: t-1}^{\prime}\right)=$ $p\left(\mathbf{z}_{t}, \mathbf{z}_{t}^{\prime} \mid \mathbf{x}_{t}, \mathbf{x}_{t}^{\prime}\right)$, and $p\left(\mathbf{x}_{t}, \mathbf{x}_{t}^{\prime} \mid \mathbf{x}_{1: t-1}, \mathbf{x}_{1: t-1}^{\prime}, \mathbf{z}_{1: t-1}, \mathbf{z}_{1: t-1}^{\prime}\right)=p\left(\mathbf{x}_{t}, \mathbf{x}_{t}^{\prime} \mid \mathbf{x}_{t-1}\right)$. In (5), we apply the properties $p\left(\mathbf{z}_{t} \mid \mathbf{z}_{t}^{\prime}, \mathbf{x}_{t}, \mathbf{x}_{t}^{\prime}\right)=p\left(\mathbf{z}_{t} \mid \mathbf{x}_{t}\right)$ and $p\left(\mathbf{z}_{t}^{\prime} \mid \mathbf{x}_{t}, \mathbf{x}_{t}^{\prime}\right)=p\left(\mathbf{z}_{t}^{\prime} \mid \mathbf{x}_{t}^{\prime}\right)$.

According to particle filtering theory, ${ }^{19}$ the posterior density $p\left(\mathbf{x}_{1: t} \mid \mathbf{x}_{1: t}^{\prime}, \mathbf{z}_{1: t}, \mathbf{z}_{1: t}^{\prime}\right)$ can be approximated as

$$
p\left(\mathbf{x}_{1: t} \mid \mathbf{x}_{1: t}^{\prime}, \mathbf{z}_{1: t}, \mathbf{z}_{1: t}^{\prime}\right) \approx \sum_{n=1}^{N} w_{t}^{n} \delta\left(\mathbf{x}_{1: t}-\mathbf{x}_{1: t}^{n}\right),
$$

where $\left\{\mathbf{x}_{1: t}^{n}, n=1 \ldots N\right\}$ is a set of samples, $\left\{w_{t}^{n}, n=1 \ldots N\right\}$ are the associated weights, and $\delta$ is the Dirac delta function. Based on importance sampling theory, the weights can be given by

$$
w_{t}^{n} \propto p\left(\mathbf{z}_{t} \mid \mathbf{x}_{t}\right) p\left(\mathbf{z}_{t}^{\prime} \mid \mathbf{x}_{t}^{\prime}\right) p\left(\mathbf{x}_{t}^{\prime} \mid \mathbf{x}_{t}\right) w_{t-1}^{n}
$$

In (7), $p\left(\mathbf{z}_{t} \mid \mathbf{x}_{t}\right)$ and $p\left(\mathbf{z}_{t}^{\prime} \mid \mathbf{x}_{t}^{\prime}\right)$ are object observation likelihood and background observation likelihood, respectively, which can be estimated by the image observation data. In our experiments, we have used kernel-based histogram to weight the samples. Specially, we can estimate the local likelihood density as follows:

$$
p\left(\mathbf{z}_{t} \mid \mathbf{x}_{t}^{n}\right)=\frac{1}{2 \pi \sigma_{1}} \exp \left\{-\frac{\left\{h\left(\mathbf{z}_{t} \mid \mathbf{x}_{t}^{n}\right)-h_{0}\right\}^{2}}{\sigma_{1}^{2}}\right\} .
$$

where $h_{0}$ is a prior histogram model of the target object, $h\left(\mathbf{z}_{t} \mid \mathbf{x}_{t}^{n}\right)$ is the histogram of sample $\mathbf{x}_{t}^{n}$.

Similarly, we have the background likelihood density,

$$
p\left(\mathbf{z}_{t}^{\prime} \mid \mathbf{x}_{t}^{\prime n}\right)=\frac{1}{2 \pi \sigma_{2}} \exp \left\{-\frac{\left\{h^{\prime}\left(\mathbf{z}_{t} \mid \mathbf{x}_{t}^{n}\right)-h_{0}^{\prime}\right\}^{2}}{\sigma_{2}^{2}}\right\} .
$$

where $h_{0}^{\prime}$ is a prior background histogram model.

$p\left(\mathbf{x}_{t}^{\prime} \mid \mathbf{x}_{t}\right)$ is called "object-background-belt relation density". Since we use a predefined fixed width to select the background belt, in our experiments, we assume it's a Gaussian distribution. When the posterior is estimated after iterations, its expectation can be calculated to give the final estimated object shape. 


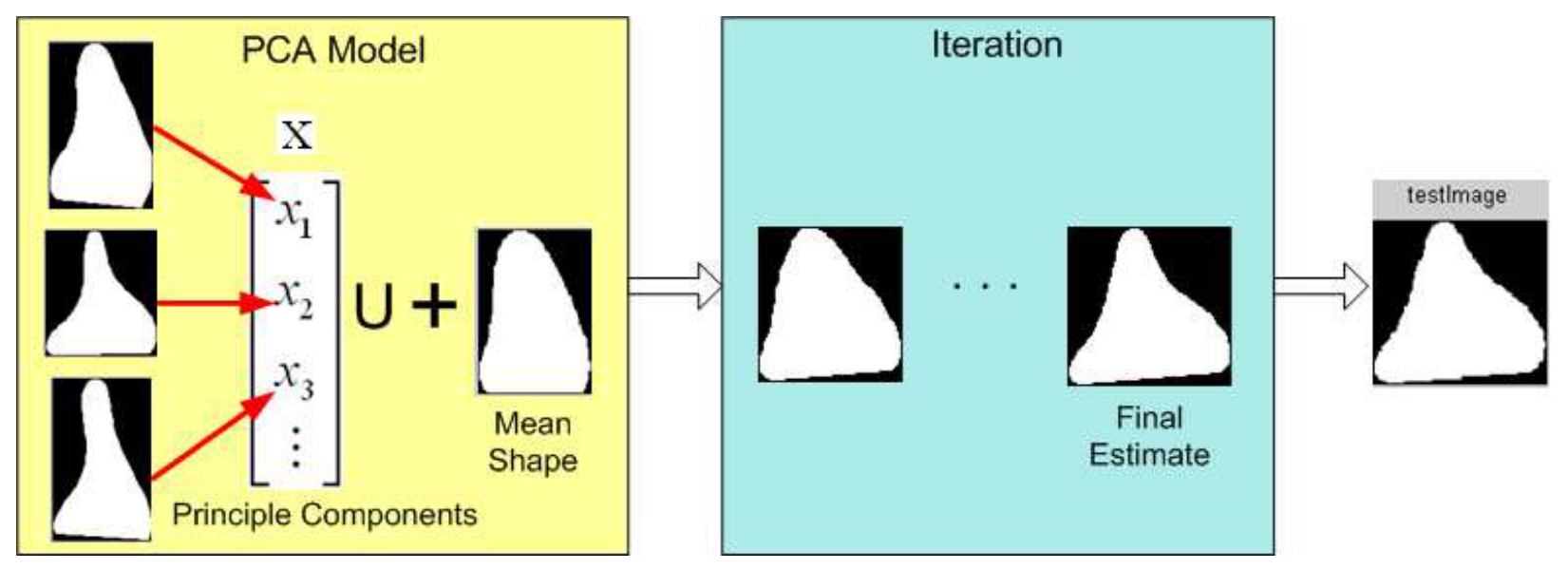

Figure 3. Conceptual demonstration of the proposed PCA-based segmentation method on synthetic data.

\section{EXPERIMENTAL RESULTS}

In order to demonstrate the performance of our proposed approach, we have tested it on both synthetic and real clinical image data. Two PCA models: one for left ventricle in 2-chamber view ultrasound echocardiography, and one for left ventricle in X-ray angiographic images were used in our experiments. The models were trained among sample shapes manually selected from thousands of medical images. $4 \sim 7$ principle components (PCs) were used in our simulation. Without code optimization, our current implementation can achieve robust segmentation within $1 \sim 4$ seconds using Matlab on a regular PC with Intel Pentium IV 3.2GHz CPU and $1 \mathrm{G}$ RAM.

\subsection{Conceptual Demonstration}

We firstly use a synthetic image to test the proposed algorithm. Fig. 3 shows the main procedure. In this experiment, the PCA model of the left ventricle in 2-chamber view ultrasound images was used. Seven PCs were used to generate the test target image as shown on the right in Fig. 3. Then only five PCs were used for estimation in order to save the converging time. A mean shape was used for initialization. After 46 iterations, the algorithm converged at the final estimate. As we can see, it is very similar to the test target image.

\subsection{Real Clinical Data}

We have applied the proposed segmentation method on real clinical data including noisy X-ray angiographical image data and ultrasound image data. An image preprocessing step was adopted to roughly find the desirable object's center. The particle filter's state vector was also extended to include the object's location information. Through this way, we can achieve more robust segmentation, which allows not only shape change but also position change for the samples. Fig. 4 illustrates some segmentation results on noisy medical image data. Row (a) to (c) are X-ray left ventricular angiographical images. Row (d) shows the results of our approach on ultrasound images. As we can see, comparing with the results in Fig. 1 which used snake and graph cut segmentation approaches, our algorithm achieved far superior performance. Due to exploiting the prior shape information inherent in the trained PCA model, the proposed method avoids the leakage problem which most graph cut based algorithms are suffered with on noisy medical image data. Even with much background noise, clutter, shadow, and uneven distributed contrast in the X-ray left ventricular or ultrasound images, the proposed approach still could achieve very robust segmentation results. 

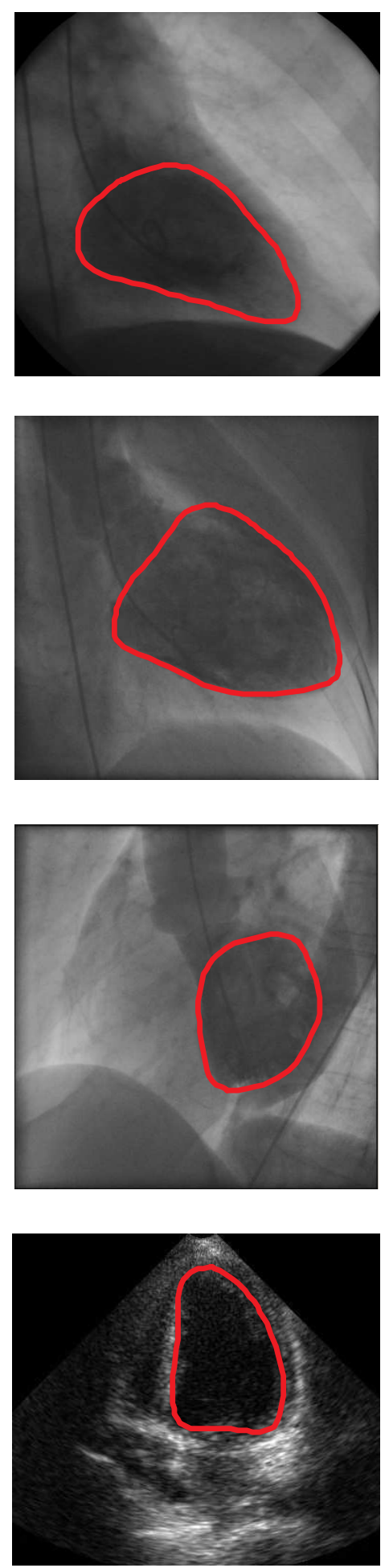

(d)
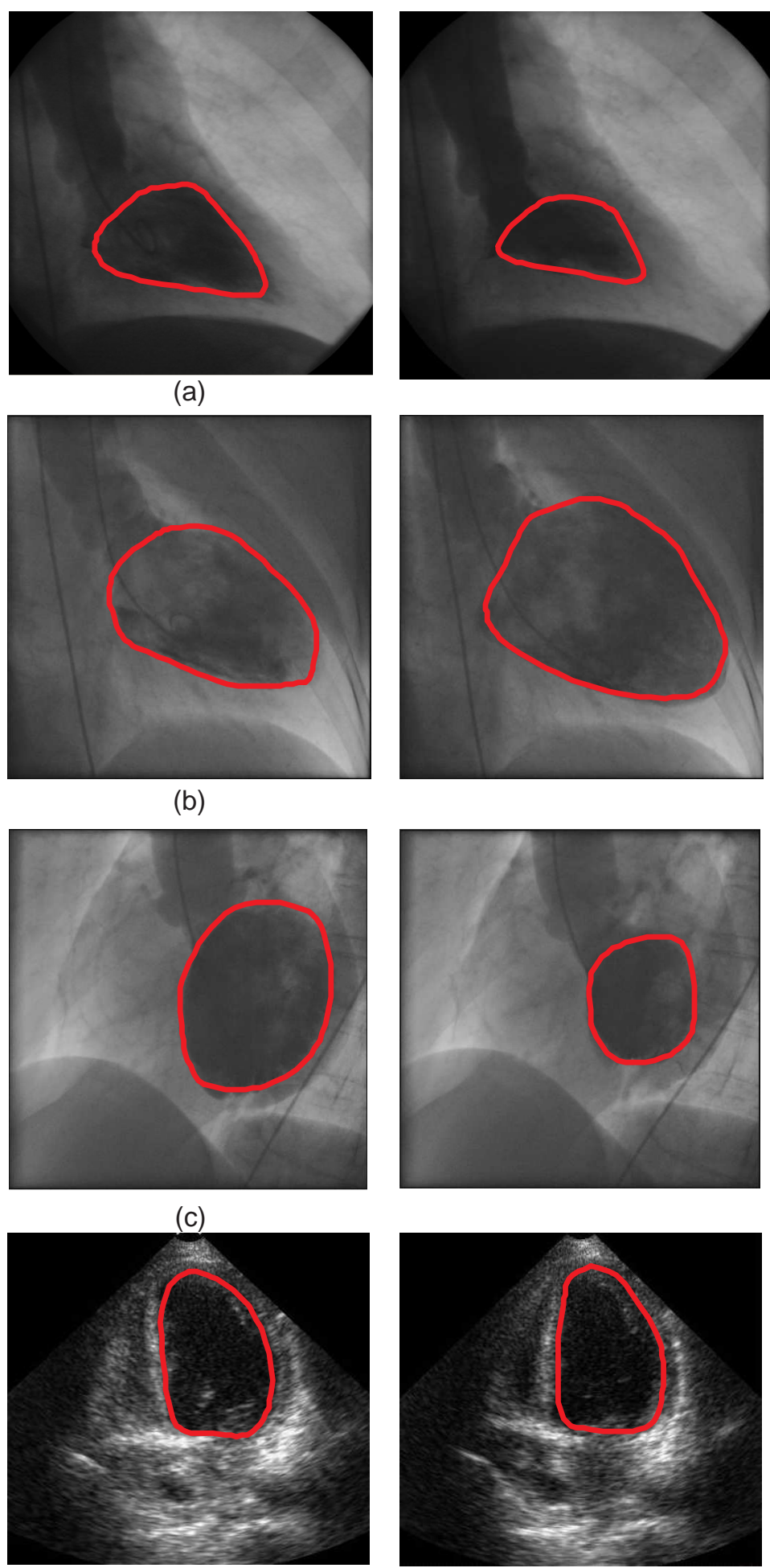

(a)

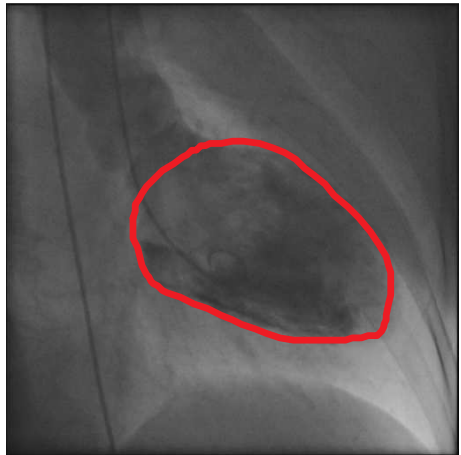

(b)
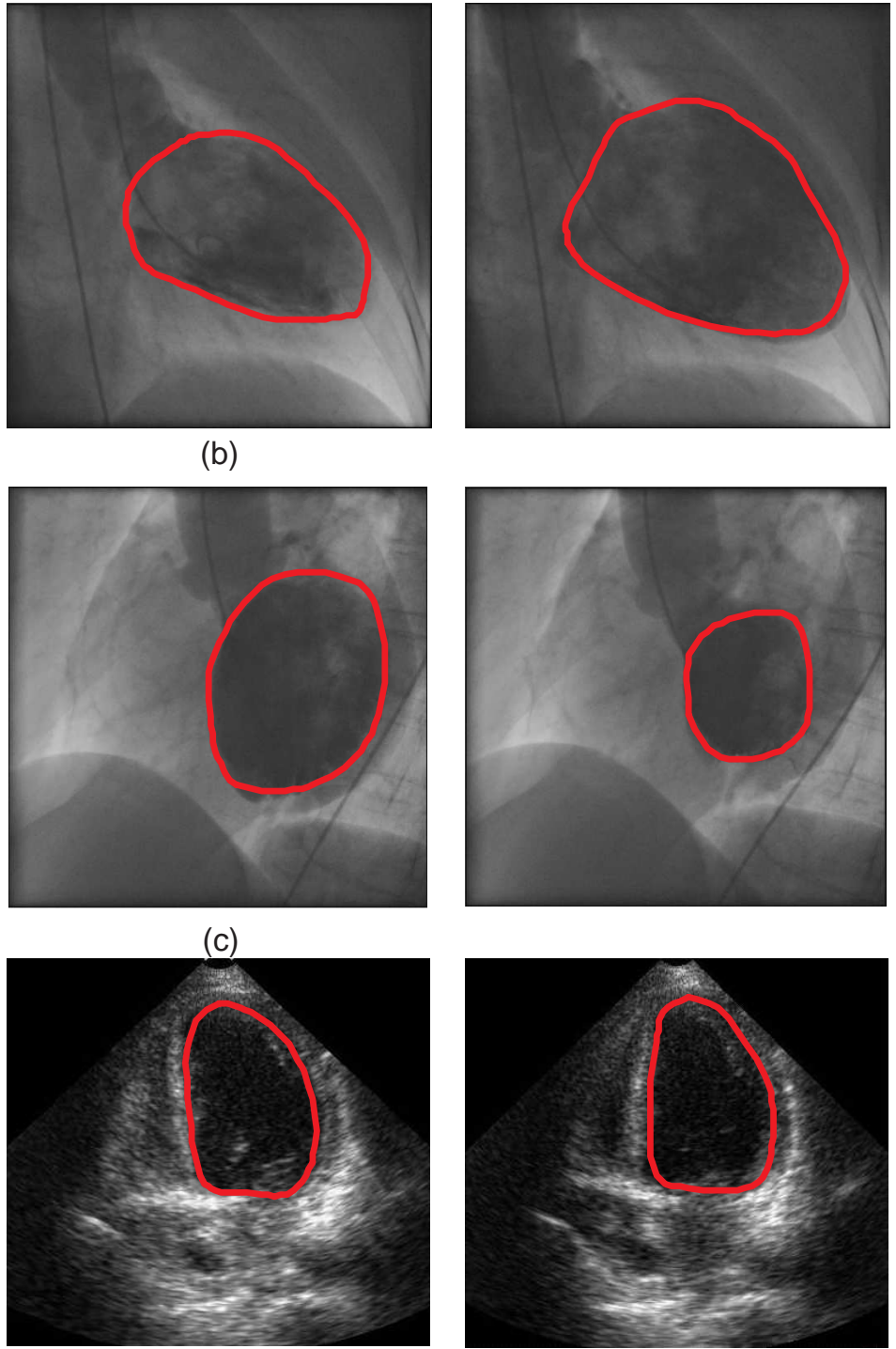

Figure 4. The segmentation results for real clinical data. (a), (b), and (c) are X-ray left ventricular angiographical images; (d) is ultrasound image data. 


\section{CONCLUSIONS}

This paper presents a novel segmentation framework in noisy medical images using PCA model based particle filtering. It exploits the prior clinical knowledge of object's shape information through a PCA model. Particle filter is further used to sample the shape space, evaluate the likelihood of each sample shape given observed image data, and then estimate the object shape by weighted combination of all shape samples. The preliminary results have demonstrated the superior performance of our approach comparing with other existing segmentation methods on both synthetic and real clinical data. For the future work, we plan to test our algorithm on more clinical applications. We are also expecting to make a real-time implementation with $\mathrm{C} / \mathrm{C}++$.

\section{REFERENCES}

1. B. Manjunath and R. Chellapa, "Unsupervised texture segmentation using markov random field models," IEEE Trans. on Pattern Analysis and Machine Intelligence 13, pp. 478-482, 1991.

2. M. Kass, A. Witkin, and D. Terzopoulos, "Snakes: Active contour models," International Journal of Computer Vision 1, pp. 321-331, 1987.

3. V. Caselles, R. Kimmel, and G. Sapiro, "Geodesic active contours," in Proc. of IEEE Int'l Conf. on Computer Vision, pp. 694-699, 1995.

4. R. Malladi, J. Sethian, and B. Vemuri, "Shape modeling with front propagation: A level set approach," IEEE Trans. on Pattern Analysis and Machine Intelligence 17(2), pp. 158-175, 1995.

5. J. Shi and J. Malik, "Normalized cuts and image segmentation," IEEE Transactions on Pattern Analysis and Machine Intelligence 22(8), pp. 888-905, 2000.

6. Y. Boykov, O. Veksler, and R. Zabih, "Fast approximate energy minimization via graph cuts," IEEE Transactions on Pattern Analysis and Machine Intelligence 23(11), pp. 1222-1239, 2001.

7. N. Xu, R. Bansal, and N. Ahuja, "Object segmentation using graph cuts based active contours," in Proc. of IEEE International Conference on Computer Vision and Pattern Recognition, 2003.

8. D. Comaniciu and P. Meer, "Mean shift: A robust approach toward feature space analysis," IEEE Trans. on Pattern Analysis and Machine Intelligence 24(5), pp. 603-619, 2002.

9. L. H. Staib and J. S. Duncan, "Boundary finding with parametrically deformable models," IEEE Trans. on Pattern Analysis and Machine Intelligence 14(11), pp. 1061-1075, 1992.

10. D. Metaxas, Physics-Based Deformable Models, Kluwer Academic Publishers, 1996.

11. X. Huang, D. Metaxas, and T. Chen, "Metamorphs: Deformable shape and texture models," in Proc. of IEEE Conf. on Computer Vision and Pattern Recognition, 1, pp. 496-503, 2004.

12. L. A. Vese and T. F. Chan, "A multiphase level set framework for image segmentation using the Mumford and Shah model," Int'l Journal of Computer Vision 50(3), pp. 271-293, 2002.

13. S. Zhu and A. Yuille, "Region Competition: Unifying snakes, region growing, and Bayes/MDL for multi-band image segmentation," IEEE Trans. on Pattern Analysis and Machine Intelligence 18(9), pp. 884-900, 1996.

14. N. Paragios and R. Deriche, "Geodesic active regions and level set methods for supervised texture segmentation," Int'l Journal of Computer Vision 46(3), pp. 223-247, 2002.

15. T. F. Cootes, C. J. Taylor, D. H. Cooper, and J. Graham, "Active shape models - their training and application," Computer Vision and Image Understanding 61(1), pp. 38-59, 1995.

16. X. Huang, N. Paragios, and D. Metaxas, "Shape registration in implicit spaces using information theory and free form deformations," IEEE Transactions on Pattern Analysis and Machine Intelligence 28(8), pp. 1303-1318, 2006.

17. X. Huang, N. Paragios, and D. Metaxas, "Establishing local correspondences towards compact representations of anatomical structures," in International Conf. on Medical Image Computing and Computer Assisted Intervention, (926-934), 2003.

18. M. E. Leventon, E. L. Grimson, and O. Faugeras, "Statistical shape influence in geodesic active contours," in Proc. of IEEE Conf. on Computer Vision and Pattern Recognition, 1, pp. 1316-1323, 2000.

19. M. S. Arulampalam, S. Maskell, N. Gordon, and T. Clapp, "A tutorial on particle filters for online nonlinear/nonGaussian Baysian tracking," IEEE Trans. Signal Processing 50, pp. 174-188, Feb. 2002.

20. W. Qu, D. Schonfeld, and M. Mohamed, "Real-time interactively distributed multi-object tracking using a magneticinertia potential model," IEEE Transactions on Multimedia 9(3), pp. 511-519, 2007. 